\title{
Synthesis, Characterisation and Photocatalytic Activity of Cadmium Sulphide Nanoparticles
}

\author{
SACHIN B. KAKODKAR \\ Department of Chemistry, Parvatibai Chowgule College of Arts and Science (Autonomous), \\ Gogol-Margao, Goa-403602, India \\ sbk001@chowgules.ac.in
}

Received 9 October 2015 / Accepted 28 October 2015

\begin{abstract}
Cadmium sulphide (CdS) nanoparticles were synthesized using thiourea as a source for sulphide ion in the presence of disodium succinate. The prepared nanoparticles were characterized by IR spectroscopy, $x$-ray diffraction and scanning electron microscopy. The photocatalytic activity of CdS was tested for degradation of methylene blue and rhodamine B. CdS catalyst shows high activity for the photocatalytic degradation of methylene blue dye.
\end{abstract}

Keywords: Cadmium sulphide, IR spectra, Photocatalytic activity, SEM, X-ray diffraction

\section{Introduction}

Photocatalytic processes on semiconductor surfaces have gained increasing importance in recent times with significant applications in areas of environment, energy production and organic reactions. Cadmium sulphide and lead sulphide are important semiconductors possessing optical and electronic properties. Cadmium sulphide ${ }^{1-4}$ is being increasingly used as catalysts for various photocatalytic reactions. The method of synthesis is found to have a pronounced effect on the catalytic activity of CdS. In the present investigation CdS has been synthesized by in presence of disodium succinate and its photocatalytic activity was tested for degradation of methylene blue and rhodamine B.

\section{Experimental}

$20 \mathrm{mmol}$ of $\mathrm{CdCl}_{2} \cdot \mathrm{H}_{2} \mathrm{O}$ and $20 \mathrm{mmol}$ of thiourea was weighed and made up to $150 \mathrm{~mL}$ using distilled water. The solution was heated for 5 minutes and $20 \mathrm{mmol}$ of disodium succinate was added to it. The solution was further heated for $4 \mathrm{~h}$. The solution was filtered and the solid was repeatedly washed with double distilled water and dried in an oven at $60{ }^{\circ} \mathrm{C}$. The resulting yellow solid $(\mathrm{CdS})$ was finely powdered using an agate mortar and pestle.

\section{Characterisation methods}

IR spectra of samples were recorded using Shimadzu IRPrestige-21 FTIR in the range of $4000-400 \mathrm{~cm}^{-1}$. The $\mathrm{x}$-ray diffraction patterns of samples were recorded using Rigaku in $2 \theta$ 
range of $5^{\circ}$ to $60^{\circ}$. The SEM micrographs of the samples were recorded using JSM-5800LV scanning electron microscope.

\section{Photocatalytic activity}

The photocatalytic activity of $\mathrm{CdS}$ and $\mathrm{PbS}$ were tested for the degradation of methylene blue and rhodamine B dyes.

\section{Photocatalytic degradation of methylene blue $(M B)$ and rhodamine $B(R h B)$}

The photocatalytic reaction was carried out with $0.1 \mathrm{~g}$ of catalyst suspended in $100 \mathrm{~mL}$ of dye solutions in a $150 \mathrm{~mL}$ beaker and then the solution was exposed to a $200 \mathrm{~W}$ tungsten lamp. The initial concentrations of $\mathrm{MB}$ and $\mathrm{RhB}$ were $50 \mu \mathrm{mol} / \mathrm{L}$. At definite time intervals, an aliquot of the dye solution were withdrawn from the reaction vessel and absorbance was measured using a Shimadzu mini $1240 \mathrm{UV}$-Vis Spectrophotometer at a wavelength of $650 \mathrm{~nm}$ and $500 \mathrm{~nm}$ for $\mathrm{MB}$ and $\mathrm{RhB}$ respectively.

\section{Results and Discussion}

Figure 1 shows IR spectrum of CdS.

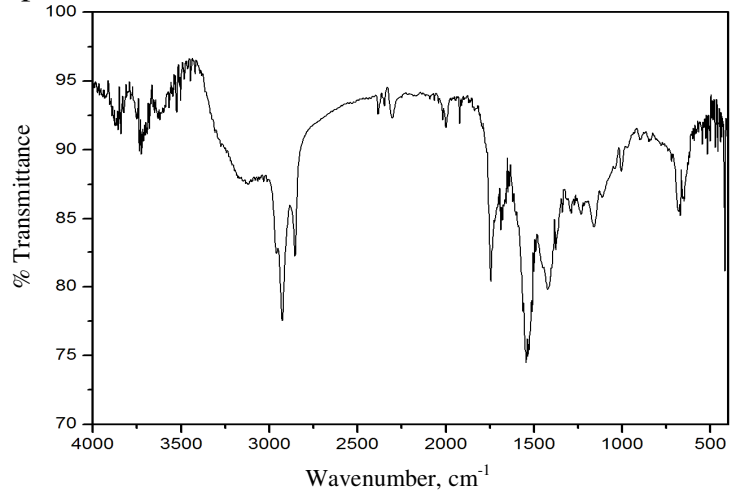

Figure 1. IR spectrum of $\mathrm{CdS}$

The bands at $650 \mathrm{~cm}^{-1}$ and $730 \mathrm{~cm}^{-1}$ correspond to Cd-S stretching band. The bands at 1620 $\mathrm{cm}^{-1}$ and $3550 \mathrm{~cm}^{-1}$ are attributed to bending vibration of water and band at to O-H stretching of adsorbed water on surface of CdS. Figure 2 shows x-ray diffraction pattern of CdS.

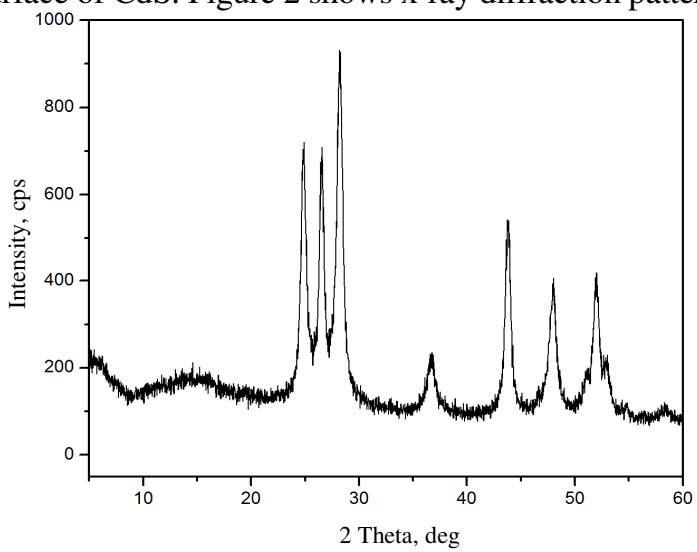

Figure 2. $\mathrm{X}$ ray diffraction pattern of $\mathrm{CdS}$ 
The pattern can be indexed to hexagonal cadmium sulfide phase and no peaks due to impurities are observed. From the Full Width at Half Maximum (FWHM) of the most intense peak the particle size has been calculated using Scherrer formula, $D=k \lambda / \beta \cos \theta$, where $\lambda$ is the wavelength of $\mathrm{x}$-ray radiation $(\mathrm{CuK} \alpha$ radiation with $=0.154 \mathrm{~nm}), \beta$ is the FWHM of the XRD peak in radians and $\theta$ is the angle of diffraction. The particle size of the sample is found to be $15 \mathrm{~nm}$.

\section{Scanning electron microscopy studies}

The SEM micrograph of CdS is shown in Figure 3.

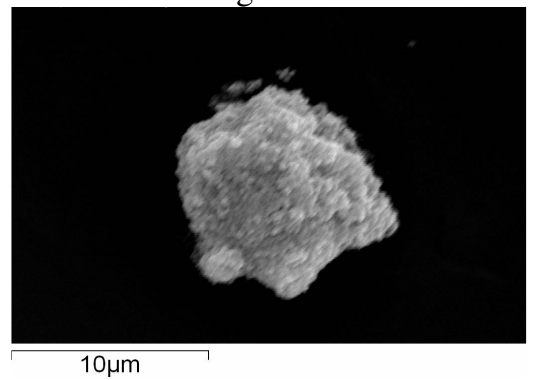

Figure 3. SEM micrographs of CdS

The CdS particles appear highly agglomerated in nature. The particles exhibit irregular morphology with particle size found to be in nano range. The agglomeration may be induced during the crystal growth itself because of the small size regime of particles as evident from XRD analysis.

\section{Photocatalytic Activity}

The photocatalytic activity of $\mathrm{CdS}$ for degradation of methylene blue and rhodamine $\mathrm{B}$ is shown in Figure 4.

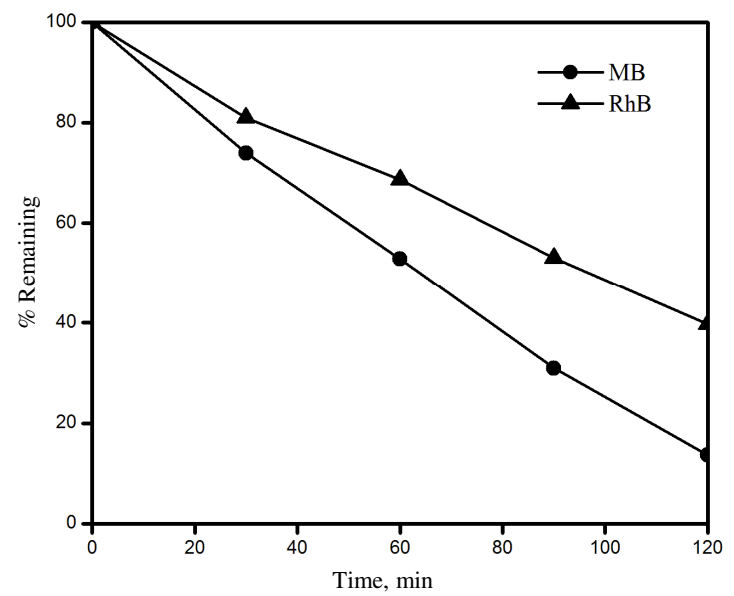

Figure 4. Photocatalytic activity of $\mathrm{CdS}$ for degradation of $\mathrm{MB}$ and $\mathrm{RhB}$

CdS catalyst showed high activity for the degradation of methylene blue (nearly $85 \%$ degradation of methylene blue was observed in 120 minutes). The catalyst showed a lower photocatalytic activity for the degradation of Rhodamine B (nearly $60 \%$ degradation of rhodamine B was observed in 120 minutes). 


\section{Conclusion}

CdS nanoparticles synthesized by using thiourea as source of sulphide ions in presence of disodium succinate showed high activity for photocatalytic degradation of methylene blue.

\section{References}

1. Warrier M, Lo M K F, Monbuquette H and Garcia-Garibay M A, Photochem Photobiol Sci., 2004, 3, 859-863; DOI:10.1039/B404268A

2. Kothari S, Kumar A, Vyas R, Ameta R and Punjabi P P, J Braz Chem Soc., 2009, 20(10), 1821-1826.

3. Giribabu K, Suresh R, Manigandan R, Vijayraj A, Prabu R and Narayanan V, Bull Korean Chem Soc., 2012, 33(9), 2910-2916; DOI:10.5012/bkcs.2012.33.9.2910

4. Yu Y, Ding Y, Zuo S and Liu J, Int J Photoenergy, 2011, 1; Article ID 762929, 5 pages DOI:10.1155/2011/762929 